This is the peer reviewed version of the following article: Mancell, Sara, Meyer, Rosan, Hind, Jonathan and Halter, Mary (2020) Factors impacting on eating in pediatric intestinal-transplant recipients : a mixed methods study. Nutrition in Clinical Practice, 35(5), pp. 919-926, which has been published in final form at https://doi.org/10.1002/ncp.10439. This article may be used for non-commercial purposes in accordance with Wiley Terms and Conditions for Use of Self-Archived Versions. 


\title{
Factors impacting on eating in paediatric intestinal transplant recipients: a mixed methods study
}

\begin{abstract}
Background: No empirical data are found examining why eating may be difficult for some children following intestinal transplant and not others. This study aimed to describe the eating behaviours and nutritional intake of intestinal transplant recipient children and examine factors that may impact on their eating.
\end{abstract}

Methods: Caregivers of all $(n=34)$ intestinal transplant recipients $<18$ years in the United Kingdom were invited to participate in this mixed methods study comprising a quantitative component (questionnaires, food diary) and qualitative interview. Questionnaires included the Children's Eating Behaviour Questionnaire and demographic/nutrition-related items and a three-day food diary. Analysis was by descriptive statistics using SPSS. Semi-structured telephone interviews explored caregiver perceptions of their child's eating. Analysis was thematic.

Results: Nine caregivers were recruited and completed the questionnaire and food diary. Eight of these were interviewed. Home tube feeding was required by $77 \%(n=7)$ of children post-transplant, $56 \%(n=5)$ were 'food avoidant' and median energy intake was 93\% (range, $61-137 \%)$ of requirements. The findings revealed complex, inter-related positive and negative medical, caregiver and child-related influences on eating. Learning to eat at the recommended age and having positive and significant pre-transplant eating experiences appeared protective, whilst being nil-by-mouth and having aversive experiences were barriers.

Conclusion: This study provides the first empirical evidence of factors that may impact on eating after intestinal transplant in children. The findings suggest promoting eating pre-transplant, where 
the negative physical consequences can be managed, may be protective and there may be eating difficulty predictors that could be used to facilitate targeted interventions.

\section{Introduction}

Intestinal transplant is an accepted treatment for children with intestinal failure, where parenteral nutrition (PN) cannot be weaned due to insufficient functioning gut mass. ${ }^{1}$ Transplant may be indicated where intestinal failure is deemed irreversible and there are severe, life threatening complications of PN such as liver disease, severe sepsis or limited venous access and where a very poor quality of life may be correctable through transplantation. ${ }^{2}$ Patients are assessed for their suitability for transplant by an intestinal transplant centre, a process which includes fully informing patients/parents of the risks and benefits of transplant. ${ }^{2}$ As well as being a life-saving treatment, intestinal transplant offers the possibility of a life free from PN and ideally the possibility of meeting nutrient requirements through eating and drinking. Anecdotally, this is often a strong driver for children (and their families), the majority of whom will have spent long periods in hospital and at home on PN, unable or unwilling to eat due to the physical consequences such as diarrhoea, pain and vomiting. While the majority are able to wean off $\mathrm{PN},{ }^{3}$ many children may have eating difficulties after transplant; just under half are reported to continue to require tube feeding two years post-transplant ${ }^{4,5}$ due to being unable to consume sufficient nutrition orally.

In the general population, eating difficulties have been described as causing considerable distress both for children and families. ${ }^{6-9}$ Selective eating has been associated with symptoms of depression and anxiety ${ }^{10}$ and eating difficulties may impact on a child's ability to participate in social eating, ${ }^{11}$ missing an opportunity for social integration. ${ }^{12}$ For children with intestinal failure, eating (or not) may have a significant impact on quality of life, ${ }^{13}$ an area which has become increasingly important as intestinal transplant survival rates improve. ${ }^{14,15,16}$ 
Although post intestinal transplant eating difficulties have been reported in expert opinion publications ${ }^{1,5,17,18}$ no studies have used a tool to classify eating behaviours, only two studies have reported nutritional intake following transplant ${ }^{4,19}$ and there is no empirical evidence regarding the factors that may contribute to eating difficulties. An understanding of this could help identify eating difficulty predictors, allow for pre-emptive targeted interventions and help manage caregiver expectations of post-transplant eating. The aim of this study was to describe the eating behaviours and nutritional intake of intestinal transplant recipient children and examine factors that may impact on their eating.

\section{Methods}

\section{Design}

This mixed methods sequential explanatory study ${ }^{20}$ consisted of quantitative self-completion questionnaires and a three-day food diary followed by qualitative semi-structured telephone interviews. The purpose of the qualitative component was to help explain quantitative findings ${ }^{20}$ and provide in-depth description not possible with quantitative approaches. ${ }^{21}$ This study was carried out at the two centres performing paediatric intestinal transplant in the United Kingdom (UK). Research ethics approval was granted from the Health Research Authority. All children (via their parent/guardian) from these two centres that had an intestinal transplant, were under 18 years of age and resident in the UK were invited to take part in the study $(n=34)$ as total population sampling is recommended for small, heterogeneous groups. ${ }^{22}$

Recruitment was a two-staged approach from patients identified and sent a study pack by the dietitian for each of the transplant services. The first stage included a questionnaire and a semiquantitative food diary and those that responded with this information and returned an expression of interest form for an interview were contacted in the second stage of the study. Implied consent was demonstrated through return of the questionnaires ${ }^{23}$ and food diary while for the interview verbal 
informed consent was obtained and recorded. Data collection took place between May and August 2017.

\section{Data collection}

The questionnaires completed by caregivers included 26 demographic and nutrition-related items and the Children's Eating Behaviour Questionnaire (CEBQ) ${ }^{24}$ (Supporting information Table S1) The CEBQ uses Likert scales ('never' [score one] to 'always' [score five]) across 35 items to assess the frequency of a range of eating behaviours and consists of eight subscales related to 'food approach' or 'food avoidant' behaviours. The CEBQ was completed by caregivers rather than the child to avoid potential comprehension difficulties ${ }^{25}$ while the food diary could be completed by children or caregivers or jointly with young children. It asked for the type and quantity of food and drink consumed over three days (two weekdays, one weekend day) to be recorded (Supporting information Table S2). Telephone interviews using a topic guide (Supporting information Table S3), developed iteratively, explored caregiver perceptions of their child's eating and were used to clarify any information in the food diaries or questionnaires to improve accuracy of analysis. Consent was obtained to check certain items from the questionnaire such as height and weight with the dietitian to further improve accuracy.

\section{Data analysis}

Descriptive statistics were reported with mean, standard deviation (SD), median and range depending on the assumptions of the data and analysed using SPSS version 22 (IBM Corp., Armonk, NY, USA). Associations were analysed using 2-tailed Fisher exact test for categorical data and Spearman's rank order correlation for ordinal data with $\mathrm{p}<0.05$ considered significant. CEBQ subscales with strong positive correlations $(\geq 0.60)^{26}$ were combined to categorise behaviour as 'food approach' or 'food avoidant ${ }^{\text {'27 }}$ and Cronbach's alpha measured internal consistency of responses. Weight, height and body mass index were presented as z-scores. ${ }^{28}$ Dietary information 
from the food diary and interview (as applicable) was entered into Nutritics Professional version 4.315 (Nutritics Ltd., Dublin, Ireland). Where required, information on branded foods was obtained from manufacturer and supermarket websites. Average intake was presented as a percentage of the recommended nutrient intake (RNI) or estimated average requirement (EAR). ${ }^{29}$

For the qualitative data, following verbatim transcription, transcripts were sent to participants for member-validation and interviews were thematically analysed through reading, re-reading and discussion to develop a coding structure and themes. ${ }^{30}$ Qualitative software F4analyse version 2.2 (GmbH, Marburg, Germany) was used for this process and themes were illustrated with relevant quotes. Taking a reflexive approach, ${ }^{30}$ post-interview reflections were written and the authors met regularly and discussed potential personal influences on data interpretation.

The quantitative and qualitative data were considered together to identify common findings and as part of a synergistic approach ${ }^{31}$ the way in which the findings interacted was considered.

\section{Results}

\section{Participants}

Nine caregivers were recruited and completed the questionnaire and food diary. From the nine, eight $(89 \%)$ caregivers were interviewed. Participant characteristics are shown in Table 1.

\section{Nutrient intake}

Of the nine respondents, two were discharged on an exclusive oral diet following transplant, three weaned off tube feeding (after 1-5 years) while the remainder continued to require tube feeding following transplant (0.5-8 years). Percentage median energy intake was slightly below the EAR (93\%) while median protein intake was more than double the RNI (237\%). Median intakes of all micronutrients (except Vitamin D) were above the RNI although ranges crossed to lower than 
recommended for many nutrients. Table 2 shows the combined intake from food and drink, tube feeding (and mineral and vitamin supplements where applicable).

\section{Eating behaviours}

The highest mean (SD) CEBQ scores were 3.72 (0.97) slowness in eating, 3.64 (1.35) enjoyment of food and 3.42 (1.15) food fussiness. (Table 3) There was a strong, positive, statistically significant correlation between food responsiveness and enjoyment of food $\left(r_{s}=.72, p=0.028\right)$ and slowness in eating and both food fussiness $\left(r_{s}=.76, p=0.018\right)$ and satiety responsiveness $\left(r_{s}=.71, p=0.031\right)$. Internal consistency of responses to items within each subscale was high $(\alpha=.77-.98)$. Mean values per participant from the included sub-scales are shown (Supporting information Table S4); 56\% $(n=5)$ had higher 'food avoidant' than 'food approach' scores.

\section{Quantitatively measured factors associated with eating after transplant}

Transitioning to an oral diet without the need for home tube feeding following transplant was statistically significantly associated with complementary foods being introduced at the recommended age $(p=0.028)$, learning to eat $(p=0.028)$, having significant practice eating $(p=0.028)$ and having positive pre-transplant eating experiences $(\mathrm{p}=0.028)$. Associations with these and other participant characteristics are shown (see Supporting information Table S5).

\section{Qualitatively reported factors associated with eating after transplant}

Caregiver participants reported a range of factors, grouped thematically here into those described as medical, caregiver and child-centred factors.

Medical factors were described widely as negative experiences such as diarrhoea and vomiting associated with their child's medical condition that were perceived to have influenced eating due to discomfort and fear. 
"He's very scared now to eat. He likes to eat but he's scared of vomit. Because...he can understand that when he eats he will vomit. And he stopped taking anything by...mouth now." (Participant 28)

Being nil-by-mouth was also thought to impact on the opportunity to eat- potentially preventing children from trying different tastes and textures, learning how to swallow and understanding hunger feelings. Several participants felt these interruptions, particularly in the weaning period, were detrimental.

“Some children ...they've gone through the process as a baby of exploring things...taste, textures and all that. They've got that memory and perhaps have more of a driver to get back to that enjoyment. Whereas she's never known it." (Participant 2)

Caregivers around the child were also described as having been or potentially influential in the child's eating, particularly in encouraging the child to eat as a way of enabling a more normal life, essentially allowing children to eat for pleasure rather than nutrition. Several participants felt it was important to advocate for this with the medical team:

“...I just kept on to the consultants... if it doesn't matter whether she eats or not, just let her eat...if it's not harming her...I don't care about it coming out of her backside- like gushes and gushes of water. I can deal with that...I just kept on saying that eating is so important." (Participant 33)

Socialising and interacting with food was also considered important, with some participants describing the child seeing others eating as helping children view food as 'safe' to try. Similarly, grocery shopping and food preparation were viewed as helpful to increase food familiarity, foster a healthy relationship with food and promote inclusion. 
"It had helped her...to have that good relationship with food. To recognise mealtimes. To sit down... as a family and have a meal...she never had an issue with suddenly being able to eat food post-transplant." (Participant 20)

A number of participants also felt that their child's own drive to eat was influential:

"...she would be constantly...pointing at the cupboard...wanting to eat... Because she had that WANT to eat I suppose..." (Participant 33)

Some participants felt a 'grazing' style allowed their child to control not only the food type, but the portion size and pace of the meal. Despite describing their children as enjoying eating, some participants explained that this only extended to preferred or 'safe' foods, with children choosing to have nothing if these were not available.

"She won't try a different brand...It's got to be that one or else she won't eat it..." (Participant 30)

\section{Inter-relationship of factors associated with eating after transplant}

The narratives around how post-transplant eating was influenced by medical issues, caregivers and the child themselves provided in-depth explanations for CEBQ responses and pointed to an interrelationship between factors. For example, medical issues seemed to impact directly on the child, and the child and caregiver were thought to impact on medical issues. Avoidant behaviours such as slowness in eating were viewed in some cases as resulting from a child's need for control, which some participants felt related to medical issues such as vomiting causing a fear of food. In contrast, 'food approach' behaviours such as food enjoyment were thought to relate to caregiver encouragement and engagement in social eating, which could impact on the extent to which 
children had positive pre-transplant eating experiences. A representation of the inter-relationship between factors is shown. (Figure 1)

\section{Discussion}

The purpose of this study was to examine factors that may influence eating following intestinal transplant. Many participants were receiving less than recommended levels of a number of key nutrients. From the cohort only two out of nine children were discharged on an exclusive oral diet after transplant and more participants were considered to show negative responses to food ('food avoidant') than positive responses ('food approach'). The small-scale quantitative findings suggested an association between pre- and post-transplant eating, a finding supported by qualitative data and explained in relation to three themes: medical, caregiver and child-related influences. The factors that emerged as having a positive influence included: having complementary foods at the recommended age (around six months), significant and positive pre-transplant eating experiences, caregiver encouragement and advocacy to eat, participation in social eating activities and the child's drive to eat. Negative factors included being nil-by-mouth and having aversive eating experiences.

In the context of the expert opinion intestinal transplant literature and studies of eating behaviour in either healthy children or those with other health problems, many of the findings may appear unsurprising. Study participants were of short stature (common following long-term PN), ${ }^{3} \mathrm{PN}$ was weaned within two months, ${ }^{3,28}$ many children were slow or unable to transition from tube feeding to an oral diet following transplant ${ }^{4,5}$ and were food avoidant ${ }^{1,5,17,18}$. Eating difficulties have been demonstrated in healthy children following a delay in lumpy textures, ${ }^{32}$ tube fed infants with limited food exposure, ${ }^{33}$ and paediatric intensive care survivors ${ }^{34}$ while an association with repeated food exposure and acceptance has been widely described. ${ }^{35,36}$ Eating difficulties have been associated with aversive experiences such as reflux and vomiting in those with allergies ${ }^{37}$ and eosinophilic disease, ${ }^{38}$ and with the frequency or severity of aversive experiences. ${ }^{39}$ Eating behaviours may 
change as children age ${ }^{40}$ with food neophobia most commonly seen between two and six years ${ }^{41}$ and caregivers have previously been described as influential on eating ${ }^{13}$ with control over food choices gradually shifting from parents to children with time. ${ }^{24,25}$

However, this study also provides unexpected insights from nutritional data, the CEBQ and caregivers' perspectives, as well as the means to draw those together. In particular, the fact that percentage median energy intake was below the EAR was not only unexpected given previous literature where higher intakes $(116-215 \%)^{4,19}$ have been reported and are thought to be required to compensate for inefficient absorption post-transplant, but also concerning, given that many of the children appeared to have unresolved eating difficulties as well as continuing tube feeding. Micronutrient deficiencies, commonly seen in intestinal transplant and thought to be related to intestinal malabsorption, ${ }^{42}$ may also be due to inadequate dietary intake as shown in this study. This underlines the importance of laboratory monitoring and supplementation after intestinal transplant, ${ }^{42,43}$ particularly of vitamin D in winter months. ${ }^{44}$ Children enjoying eating (even where enjoyment was limited to a few 'safe' foods or where eating was only for pleasure rather than nutrition) has not previously been described in the intestinal transplant literature. It is important in light of descriptive evidence that, where nil-by-mouth periods are unavoidable, techniques such as sham-feeding in children with oesophageal atresia ${ }^{45}$ and non-nutritive sucking in premature infants, ${ }^{46}$ as well as early food introduction and promoting eating when on $\mathrm{PN}^{1,5,47-49}$ may be ways of preventing eating difficulties.

Given that all of the children developed intestinal failure in early childhood- a stage in life where the caregiver's role is key ${ }^{50}$ - the novel finding in this study of the potential positive influence that caregivers could have on medical team decisions relating to being nil-by-mouth may be significant. This is especially relevant for children with intestinal failure given the suggestion that caregiver influence might occur most often in children with chronic illness where there is some degree of 
uncertainty about the best plan of action. ${ }^{51}$ Anecdotally, symptoms of intestinal failure such as diarrhoea may be managed in a number of different ways; for example, stopping, reducing or allowing oral intake (with careful fluid and electrolyte replacement). It may be that in some circumstances, caregivers could increase the opportunity to practice eating, alongside medical amelioration of otherwise aversive symptoms and thereby impact on eating following transplant. It is interesting to note that the pattern of eating difficulties seen in the general population did not appear to differ in transplant recipient children. However, the caregiver reports in our study highlight that many of these factors are considered vitally important for the child's post-transplant relationship with food, and their fit into societal norms related to eating. Additionally, participant narratives around children's struggles with eating after transplant and the view that difficulties could be related to the lack of control experienced through living with intestinal failure, alongside evidence of their continuing nutrient deficiencies, accentuates the impact of some potentially amenable factors.

The broad range of ages and time since transplant of children in this study afforded both short and long-term caregiver viewpoints of eating after intestinal transplant. The overall view from participants was that for children with eating difficulties it is a long and slow process to establish eating - in common with the general population of children with eating difficulties ${ }^{52}$ - and that eating difficulties tend to persist long after transplant. However, what was encouraging was a general sense from participants that improvements do tend to occur over time.

Although the number of patients in this study is small and may be perceived as having limited generalizability, intestinal failure is a rare disease $\mathrm{e}^{53}$ and intestinal transplant is even more rare. As such, this sample of nine patients represents a significant proportion of the total UK paediatric intestinal transplant population (over one quarter). Research into such populations is considered important and may help to advance the understanding of more common diseases. ${ }^{54} \mathrm{We}$ aimed in the 
questionnaire to collect data on the conditions, treatments and feeding regimes that we considered important in clinical practice, however it is possible that additional clinical condition details that we did not collect are important for oral tolerance, for example resection of the stomach, and should be considered in future enquires. A further limitation of the study was a reliance on caregiver-report, however several measures were included to improve the accuracy of this information and limit recall bias,${ }^{55}$ including consent from all participants to cross-check certain information with the dietitian looking after their child and clarification of information in the food diaries at the time of interview. Similarly, the interviews and CEBQ captured caregiver perceptions of their child's eating rather than the child's own perceptions, which may have differed. Despite this limitation and the fact that the CEBQ has not been validated specifically for use in the intestinal transplant population it has been shown to have good internal consistency, test-retest reliability and construct validity in different settings and with different ages. ${ }^{24,27 ; 56-59}$ This is the first study to use a tool to examine eating behaviour in this group. Furthermore, the qualitative data appeared consistent with CEBQmeasured behaviour, lessening concern about bias.

This study provides the first empirical evidence of an association between introducing complementary foods at the recommended age, positive pre-transplant eating experiences and eating after transplant. The study's findings suggest that the transition to eating may be lengthy and difficult, particularly for children who have never eaten or had negative pre-transplant eating experiences. There may be a benefit to encouraging eating and introducing foods at the normal developmental stages, where the negative physical consequences of eating, such as diarrhoea, and other aversive experiences can be managed and do not cause the child distress. These findings have relevance for intestinal failure but also for medical conditions characterised by eating disruptions in early life. Further multi-centred studies, aiming to reach a larger sample, are needed. 


\section{References}

1. Goulet $\mathrm{O}$, Ruemmele F, Lacaille F, Colomb V. Irreversible intestinal failure. J Pediatr Gastroenterol Nutr. 2004;38(3):250-269.

2. NHS Blood and Transplant. Intestinal transplantation: patient selection. 2015. Available at: https://nhsbtdbe.blob.core.windows.net/umbraco-assetscorp/4964/intestinal_selection_policy.pdf.

3. Venick RS, Wozniak LJ, Colangelo J et al. Long-term nutrition and predictors of growth and weight gain following pediatric intestinal transplantation. Transplantation 2011;92(9):1058-1062.

4. Lacaille F, Vass N, Sauvat F et al. Long-term outcome, growth and digestive function in children 2 to 18 years after intestinal transplantation. Gut. 2008;57(4):455-461.

5. Gupte GL, Beath SV. Update on intestinal rehabilitation after intestinal transplantation. Curr Opin Organ Transplant. 2009;14(3):267-273.

6. Puntis JWL. Specialist feeding clinics. Arch Dis Child. 2008;9(2):164-167.

7. Bryant-Waugh R, Markham L, Kreipe RE, Walsh T. Feeding and eating disorders in childhood. Int J Eat Disord. 2010;43(2):98-111.

8. Wu YP, Franciosi JP, Rothenberg ME, Hommel KA. Behavioral feeding problems and parenting stress in eosinophilic gastrointestinal disorders in children. Pediatr Allergy Immunol. 2012;23(8):730-735.

9. Jacobi C, Agras WS, Bryson S, Hammer LD. Behavioral validation, precursors, and concomitants of picky eating in childhood. J Am Acad Child Adolesc Psychiatry. $2003 ; 42(1): 76-84$.

10. Zucker N, Copeland W, Franz L et al. Psychological and psychosocial impairment in preschoolers with selective eating. Pediatrics. 2015;136(3):e582-e590.

11. Thompson C, Cummins S, Brown T, Kyle R. What does it mean to be a 'picky eater'? A qualitative study of food related identities and practices. Appetite. 2015;84(1):235-239. 
12. Absolom S, Roberts A. Connecting with others: the meaning of social eating as an everyday occupation for young people. J Occup Sci. 2011;18(4):339-346.

13. Hopkins J, Cermak SA, Merritt RJ. Oral feeding difficulties in children with short bowel syndrome: a narrative review. Nutr Clin Pract. 2017;20(10):1-7.

14. NHS Blood and Transplant. Annual report on intestine transplantation (2016/2017). 2017. Available at: https://nhsbtdbe.blob.core.windows.net/umbraco-assets-corp/4806/nhsbtintestinal-report-2017.pdf.

15. James C, Gupte GL. Intestinal transplantation in children: promoting quality of life as well as survival. Gastroenterol Nurs. 2010;8(4):23-28.

16. Avitzur Y, Miserachs M. Quality of life on long-term parenteral nutrition: can it be an indication for intestinal transplantation? Curr Opin Organ Transplant. 2019;23(2):199-206.

17. Silver HJ, Castellanos VH. Nutritional complications and management of intestinal transplant. J Acad Nutr Diet. 2000;100(6):680-689.

18. Weseman RA, Gilroy R. Nutrition management of small bowel transplant patients. Nutr Clin Pract. 2005;20(5):509-516.

19. Ordonez F, Barbot-Trystram, Lacaille F et al. Intestinal absorption rate in children after small intestinal transplantation. Am J Clin Nutr. 2013;97(4):743-749.

20. Creswell JW, Plano Clark VL. Designing and conducting mixed methods research. 2nd edn. 2011. London: SAGE Publications.

21. Morse JM, Niehaus L. Mixed Method Design Principles and Procedures. 2009. Walnut Creek, CA: Left Coast Press Inc.

22. Daniel J. Sampling essentials: practical guidelines for making sampling choices. 2011. London: SAGE.

23. UK Data Service. Consent for data sharing: consent in surveys 2012-2019. 2019. Available at: https://www.ukdataservice.ac.uk/manage-data/legal-ethical/consent-datasharing/surveys.aspx. 
24. Wardle J, Guthrie CA, Sanderson S, Rapoport, L. Development of the Children's Eating Behaviour Questionnaire. J Child Psychol Psychiat. 2001;42(7):963-970.

25. Ashcroft J, Semmler C, Carnell S, van Jaarsvel CHM, Wardle J. Continuity and stability of eating behaviour traits in children. Eur J Clin Nutr. 2008;62(8): 985-990.

26. Petrie A, Sabin C. Medical statistics at a glance. 3rd edn. 2009. Oxford: Wiley-Blackwell.

27. Tharner A, Jansen PW, Kiefte-de Jong JC et al. Toward an operative diagnosis of fussy/picky eating: a latent profile approach in a population-based cohort. Int J Behav Nutr Phys Act. 2014;11(1):1-11.

28. World Health Organisation. Growth reference data for 5-19 years. 2007. Available at: https://www.who.int/growthref/en/.

29. Department of Health. Dietary Reference Values for Food Energy and Nutrients for the United Kingdom: Report of the Panel on Dietary Reference Values of the Committee on Medical Aspects of Food Policy. 1991. London: HMSO.

30. Green J, Thorogood N. Qualitative methods for health research. 2009. 2nd edn. London: SAGE.

31. Hall B, Howard K. A synergistic approach: conducting mixed methods research with typological and systemic design considerations. J Mix Methods Res. 2008;2(3):248-269.

32. Coulthard H, Harris G, Emmett P. Delayed introduction of lumpy foods to children during the complementary feeding period affects child's food acceptance and feeding at 7 years of age. Matern Child Nutr. 2009;5(1), 75-85.

33. Mason SJ, Harris G, Blissett J. Tube feeding in infancy: implications for the development of normal eating and drinking skills. Dysphagia. 2005;20(1):46-61.

34. Morton K, Marino LV, Pappachan JV, Darlington AS. Feeding difficulties in young paediatric intensive care survivors: a scoping review. Clin Nutr ESPEN. 2019;30:1-9.

35. Nicklaus S. The role of food experiences during early childhood in food pleasure learning. Appetite. 2016;104:3-9. 
36. Cooke LJ, Wardle J. Age and gender differences in children's food preferences. Br J Nutr. 2005;93:741-746.

37. Meyer R, Rommel N, Van Oudenhove L, Fleming C, Dziubak R, Shah N. Feeding difficulties in children with food protein-induced gastrointestinal allergies. J Gastroenterol Hepatol. 2014;29(10):1764-1769.

38. Mukkada VA, Haas A, Creskoff Maune N et al. Feeding dysfunction in children with eosinophilic gastrointestinal diseases. Pediatrics. 2010;126(3):e672-e677.

39. Field D, Garland M, Williams K. Correlates of specific childhood feeding problems. $J$ Paediatr Child Health. 2003;39(4):299-304.

40. Dovey TM, Staples PA, Gibson EL, Halford CG. Food neophobia and 'picky/fussy' eating in children: a review. Appetite. 2008;50:181-193.

41. Cooke LJ, Wardle J, Gibson EL. Relationship between parental report of food neophobia and everyday food consumption in 2-6-year-old children. Appetite. 2003;41(2):205-206.

42. Ubesie AC, Cole CR, Nathan JD et al. Micronutrient deficiencies in pediatric and young adult intestinal transplant patients. Pediatr Transplant. 2013;17(7):638-645.

43. Vennick RS, Wozniak LJ, Colangelo J et al. Long-term nutrition and predictors of growth and weight gain following pediatric intestinal transplantation. Transplantation. 2011;92(9):1058-1062.

44. Bates B, Lennox A, Prentice A et al. The National Diet and Nutrition Survey: Results from Years 1, 2, 3 and 4 (combined) of the Rolling Programme (2008/2009 - 2011/2012). 2014. London: TSO.

45. Golonka NR, Hayashi AH. Early "sham" feeding of neonates promotes oral feeding after delayed primary repair of major congenital esophageal anomalies. Am J Surg. 2008;195(5):659-662.

46. Foster JP, Psaila K, Patterson T. Non-nutritive sucking for increasing physiologic stability and nutrition in preterm infants. Cochrane Database Syst Rev. 2017;10:CD001071. 
47. Nucci AM, Ellsworth K, Michalski A, Nagel E, Wessel J. Survey of nutrition management practices in centres for pediatric intestinal rehabilitation. Nutr Clin Pract. 2017;20(10):601606.

48. Protheroe S. Long term parenteral nutrition. Paediatr Child Health. 2015;25(9):399-405.

49. Johnson T, Sexton E. Managing children and adolescents on parenteral nutrition: challenges for the nutritional support team. Proc Nutr Soc. 2006;65(3):217-221.

50. Smith AD, Herle M, Fildes A, Cooke L, Steinsbekk S, Llewellyn CH. Food fussiness and food neophobia share a common etiology in early childhood. J Child Psychol Psychiatry. 2017;58(2):189-196.

51. Miller VA. Parent-child collaborative decision making for the management of chronic illness: a qualitative analysis. Fam Syst Health. 2009;27(3):249-266.

52. Edwards S, Davis AM, Ernst L et al. Interdisciplinary strategies for treating oral aversions in children. J Parenter Enteral Nutr. 2015;39(8):899-909.

53. Orphanet. The portal for rare diseases and orphan drugs. 2019. Available at: https://www.orpha.net/consor/cgi-bin/index.php.

54. National institute of health research. Rare disease research. 2019. Available at: https://www.nihr.ac.uk/about-us/how-we-are-managed/our-structure/infrastructure/rare$\underline{\text { diseases-translational-research-collaboration/rare-disease-research.htm. }}$

55. Bowling A. Research methods in health: investigating health and health services. $3^{\text {rd }}$ edn. 2009. Maidenhead: McGraw-Hill Open University Press.

56. Viana V, Sinde S, Saxton JC. Children's eating behaviour questionnaire: associations with BMI in Portuguese children. Br J Nutr. 2008;100(2):445-450.

57. Farrow CV, Galloway AT, Fraser K. Sibling eating behaviours and differential child feeding practices reported by parents. Appetite. 2009;52(2):307-312. 
58. De Lauzon-Guillain B, Oliveira A, Charles MA et al. A review of methods to assess parental feeding practices and preschool children's eating behavior: the need for further development of tools. J Acad Nutr Diet. 2012;112(10):1578-1602.

59. Maslin K, Grundy J, Glasbey G et al. Cow's milk exclusion diet during infancy: is there a long-term effect on children's eating behaviour and food preferences? Pediatr Allergy Immunol. 2016;27(2):141-146. 